\title{
ANATOMICAL AND EXPERIMENTAL STUDIES ON THE TEMPORAL POLE OF THE MACAQUE
}

\author{
LOUIS J. POIRIER \\ Laboratory of Comparative Neurology, Department of Anatomy, University \\ of Michigan, Ann Arbor, and Laboratoire a'histologie, \\ Université de Montreal, Montréal, Canada
}

NINETEEN FIGURES

This study dealing with the brain of the monkey is part of a research program carried on in the Laboratory of Comparative Neurology at the University of Michigan. The writer wishes to thank the A. B. Brower and E. R. Arn Research and Scholarship Fund, made available through the Dean of the Medical School, for the generous grant which made possible the present work. The author also expresses his sincere gratitude to Professor Elizabeth C. Crosby for her numerous suggestions and her unfailing cooperation.

Research on the temporal pole of the brain was suggested to the author mainly by a set of papers published by Klüver and Bucy ('37, '38, '39). After extensive removal of the temporal lobes bilaterally, these two experimenters obtained a complete syndrome, which was characterized by "psychic blindness," oral tendencies, hypersexuality, hypermetamorphosis, emotional changes, etc. Such far-reaching results from injury to this particular area of the brain appeared to the author worthy of further investigation.

\section{MATERIAL AND TECHNIQUE}

For this problem on the temporal lobe, including the amygdala, the piriform lobe, and the hippocampus, Macaca mulatta was selected. Lesions were placed in the brains of 6 monkeys

${ }^{1}$ A dissertation submitted in partial fulfillment of the requirements for the degree of Doctor of Philosophy in the University of Michigan, 1950. 
under ether anesthesia. The surface cortex was removed by electrocoagulation or with a scalpel. Deeper lesions were made chiefly by scooping out the brain tissue, and a few lesions were produced with the help of the stereotaxic instrument (the McCulloch modification). All of these monkeys except no. 6 were tested as to their ability to discriminate between the forms of various objects and between different sounds, weights, or colors.

Discrimination between various objects, including those edible and inedible, was tested according to the "multiple object tests"' as devised by Klüver and Bucy ('39). In teaching visual form discrimination, two boxes similar in size, color, and weight were used. One of the two differed from the other only by presenting along its upper edge a semilunar notch (fig. 18). In order to obtain food, the animal had to pull in the box which presented a semilunar edge. (A test of this type was first described by Klüver, '33.) Both boxes were placed at a distance of about 8 feet from the cage. When the animal was able to pull in the correct box directly for from 17 to 21 trials, the test was considered as learned. For discriminating between weight, sound, or color, the two boxes varied one from the other only by differences in weight, sound or color respectively. For discriminating between weights, food was placed in the lighter of two boxes, using weights of $200 \mathrm{gm}$ and of $50 \mathrm{gm}$ in the heavier box. For discriminating between sounds the animal, in order to obtain food, had to pull in the box which was not connected with a bell. Thus the animal discriminated between sound and quietude. So in these last two tests, he had to test the situation each time before pulling in the correct box. For color discrimination metallic gray and white were used. All these tests were learned preoperatively after an average of 150 to 200 trials.

The behavior of the animals was also recorded pre- and postoperatively by observing the monkeys in the cages and noting their reactions toward food, toward the experimenter, and toward other animals - in other words, their social be- 
havior. After various lengths of time (two to three weeks) the animals were killed by an overdose of nembutal, the tissue being perfused with $10 \%$ to $15 \%$ formalin ( $40 \%$ formaldehyde) while the heart was still beating. After removal, their brains were fixed for a few days in $10 \%$ formalin. Then they were stained with osmic acid according to the Marchi technique. In the case of the brain of monkey no. 6 , the slices of the brain tissue were placed on a thread, and arranged in an upright position in the staining fluid, as suggested by Krieg ('49) and others; the degenerated myelin was found to be well impregnated by the osmic acid. In other cases an attempt was made to restain with osmic acid some sections in which the degenerated fibers were rather pale. Individual sections were run through xylol, alcohol, and distilled water and then placed in the staining solution proposed by Swank and Davenport ('35) for slices of tissue. The fibers which had been stained weakly appeared darker and sharper after this treatment and could be photographed more easily, as shown in figure 15. Some lesions in this series of cases were older than three weeks, and for this reason some fibers showed retrograde degeneration and certain nuclear masses were filled with black granules; this was especially evident in the thalamo-cortical fibers and the thalamic nuclei (fig. 7 ).

The brain of monkey no. 1 was studied only grossly; 5 series of monkey brains with various lesions were available for microscopical studies. Microphotographs were taken with the generous help of Mr. George Smith, technician in the Laboratory of Comparative Neurology.

\section{DESCRIPTION}

\section{Monkey no. 1}

This monkey learned preoperatively to discriminate between the shape of objects by the pulling-in technique; he performed the last 50 trials without error. After only a few trials it could discriminate between various objects (screw, bottle cap, cracker, laundry pin, cigarette, eraser, cube of sugar, piece of cardboard, piece of apple) placed on a board in front 
of the cage. Following a left temporal lobotomy (November $5,1949)$, the same tests were repeated and they were all recalled perfectly. Three weeks later (November 26, 1948) a right temporal lobotomy was carried out. In the following days, the differences between the shape of various objects were recalled perfectly, and new objects (a metallic hook, a casein cube, a piece of chalk, a date) presented to the animal, one at a time, were quickly learned. Discrimination of form was nearly as good as before, the animal making only two errors out of 27 trials.

Examination of the brain at autopsy (December 2, 1948) revealed that the superior, the middle, and the inferior temporal gyri had been sectioned on both sides, the hippocampal gyrus and hippocampus being spared. On the right side the incision corresponded to a plane which continues downward from the lower end of the central fissure through the temporal lobe. On the left the incision was about $\frac{1}{2} \mathrm{~cm}$ caudal to that on the right.

Summary. After bilateral lobotomy of nearly the anterior two-thirds of the temporal lobe, but not involving the hippocampal gyrus and hippocampus, no changes in behavior were observed. Discriminatory tests were performed as well after this bilateral lesion as preoperatively and the animal learned to discriminate between new objects as rapidly as before.

\section{Monkey no. 2}

Preoperatively this monkey learned to recognize differences in the form of objects, showing no errors in the last 18 trials; it could also discriminate between various objects (see objects listed in testing monkey no. 1) placed on a board in front of it. Through an opening in the left superior temporal gyrus, a lesion was placed in the region of the uncus on January 21, 1949. Postoperative testing did not disclose any loss in the ability to recognize the previously learned objects or to carry out the earlier learned tests, and observation did not show any changes in its behavior. 
On February 4, 1949, the animal was reoperated on the right side, this lesion involving chiefly the superior, the middle, and the inferior temporal gyri. Tests for discrimination, on being repeated postoperatively, revealed only a little loss of memory for form. In 50 such trials the animal made only 8 errors. Discrimination between previously learned objects was as good as before operation but this monkey was able to learn to discriminate between new objects only after repeated trials. Although it had been very wild previous to this last operation, it was now rather tame and could be approached and handled with ease by the experimenter. An attempt was made to have the animal discriminate between weights by the pulling-in technique; however, after numerous trials it did not show any sign of learning, even when there was a difference of as much as $250 \mathrm{gm}$ in the weights. This animal was sacrificed on February 10, 1949.

Macroscopic and microscopic anatomy. Study of the brain of monkey no. 2 revealed the following destruction and degeneration. On the left side (first operation) the middle portion of the superior temporal gyrus and a little overlying. parietal cortex were destroyed. Deeper, most of the lower half of the insular cortex was involved, together with the claustrum and its two limiting capsulae, extrema and externa. The lower part of the putamen was partially affected by the lesion also. Still deeper the instrument severed the tail of the caudate nucleus and the stria terminalis; forward, the uncus and the amygdala were largely involved and also the transverse limb of the anterior commissure and the uncinate fasciculus. Very few degenerated fibers were found in the left temporo-pontine tract, but stria terminalis on this same side had degenerated fibers throughout its course. On the right side (second operation) the superior temporal gyrus showed deep destruction in the middle third of its extent and the middle temporal gyrus in its caudal half; also, a large part of the insula, about the lower two-thirds, the capsula extrema and the claustrum were involved, the capsula externa being spared. A very little of the base of the parietal cortex was 
destroyed. More medially and deeper, the right fasciculus uncinatus was sectioned and about the anterior one-third of the ventral portion of the right inferior temporal gyrus was completely destroyed.

The right temporo-pontine tract showed many more degenerated fibers than did the homologous tract on the left side (fig. 1). On the left side a large part of the superior temporal gyrus was destroyed; however, the degenerated fibers in the left temporo-pontine tract could not be traced to this cortex, for they were injured in their course through the putamen. This fact gives further support to the view that the temporopontine tracts have their origin in the middle and inferior temporal gyri (Dejerine, 1895). In this case the degenerated fibers in the right temporo-pontine tract appear to come chiefly from the right middle temporal gyrus, which was injured more caudally than the right inferior temporal gyrus (Crosby and Henderson, '48).

Summary. After a left lesion which involved the superior temporal and insular cortices, the stria terminalis, and the basal parts of the putamen, the claustrum, and the capsulae externa and extrema and which destroyed, to a large extent, the uncus and the left amygdala, this monkey showed no change in behavior and he recalled previously learned tests and objects. A second lesion on the right side destroyed parts of the superior, the middle, and the inferior temporal gyri, and a large part of the insula in addition to the claustrum and capsula extrema. After this operation the monkey was very tame and more vocal than previously. It recalled previously learned objects and tests, but when a new test (a test for discriminating between weights) was introduced, could not learn the test. The animal could learn to discriminate between new objects only after multiple attempts.

\section{Monkey no. 3}

This monkey learned preoperatively to discriminate between the form and the weight of objects by the pulling-in 
technique, making no errors in the last 27 and 21 trials, respectively. After only a few attempts the animal discriminated between various objects placed in front of the cage (screw, cracker, piece of apple, etc., as listed for monkey no. 1), picking up only those which were edible and eating them. On February 25, 1949, a first lesion was placed along the basal and medial surface of the left temporal lobe through a small opening in the superior and the middle temporal gyri. Following this first lesion, the monkey performed the discrimination tests as well as before and no changes in behavior were observed. On March 4, 1949, a similar lesion was made on the right side, following which this animal (like monkey no. 2) showed greater tameness and uttered more sounds than previously. However, as far as discrimination was concerned, the picture was quite different. Discrimination between weights was perfectly recalled and various objects placed on a board were recognized at once; new objects could be learned and selected after a few trials only. The animal could no longer discriminate by the form of an object, and was still unable to do so after 200 trials. Its performance on the multiple object tests revealed that no visual impairment was present, which could explain its loss of discrimination for form. On being tested for form, the animal shifted from one box to the other at each trial before pulling in one box; it appeared that, although it could see both boxes, it could not, so to speak, "make up its mind" as to which of the two boxes he should pull to obtain food.

A third lesion, on March 11, 1949, which destroyed the surface cortex of the left temporal lobe around the previous opening in the brain, added nothing to the last-described picture of the animal. A similar lesion on the right side made a week later (March 18, 1949) was followed by a greater loss. However, the ability to discriminate between weights was still retained, but now the animal was not only unable to discriminate between the forms of boxes but also between various objects placed on a board in front of it. The monkey picked up indiscriminately any object offered (as a metallic 
hook or a date), smelling such an object, licking it, and eating it when it was edible. However, sometimes even certain edible objects of which the animal was fond were discarded, for instance unshelled peanuts. If the experimenter placed his finger in front of the animal, the animal grasped it at once just as it seized any other object in sight. Vocalization, which was very marked following the second and third operations, disappeared completely and the tameness increased. An autopsy was performed on March 25, 1949.

Macroscopic and microscopic anatomy. Study of this brain revealed that on the left side approximately the middle third of the superior temporal gyrus and about the caudal third of the middle temporal gyrus were destroyed together with the rostral portion of area 19 and a little of the lower part of the parietal cortex. More medially and rostrally this same lesion involved the lateral olfactory tract, the prepiriform cortex, and the anterior limb of the anterior commissure; the dorsal part of the amygdala and fasciculus uncinatus were damaged and the capsulae externa and extrema and the claustrum were destroyed at the base of the brain, together with part of the transverse limb of the anterior commissure. The lesion also involved the lower part of the insula and of the putamen, interrupting some of the fibers which run through this latter structure, and included the basal surface of the inferior temporal gyrus and about the lower half of the hippocampus.

On the right side the superior temporal gyrus was destroyed in about the same area as on the left; in addition the lower part of the pre- and postcentral gyri showed degeneration. More medially and more rostrally the uncus and the amygdala were largely destroyed; the inferior temporal gyrus, especially its ventral portion, and the hippocampal gyrus were deeply disintegrated rostrocaudally together with the rostral portion of the hippocampus. Above this latter structure the stria terminalis and the tail of the caudate were encroached upon by the lesion. More laterally, the fasciculus uncinatus was separated from the temporal pole by the lesion. 
The capsulae extrema and externa showed degenerated fibers in their vertical limbs, these fibers having been destroyed at the base of the brain along with the claustrum. The transverse limb of the anterior commissure was also involved at its origin in the temporal pole. Very little of the putamen was affected but many fibers were injured just ventrolateral to this structure.

From these superficially and deeply destroyed structures degenerated fibers could be traced into both the left olfactory tracts (medial and lateral), into the right stria terminalis (fig. 2), and into both fornices. The degenerated fibers in the right stria terminalis could be followed chiefly to the bed nucleus of this tract. Fibers showing Marchi granules were more numerous in the left fornix; some of these fibers could be traced to the left lateral septal nucleus (fig. 3) and others to the ventral half of the left mammillary body. Those in the right fornix, fewer in number, could be followed only to the right lateral septal nucleus (fig. 3). Marked degeneration occurred in the left temporo-pontine tract, which was partially destroyed in the putamen together with a few fibers of the temporo-pulvinar fasciculus of Arnold and a few fibers of the optic radiations. Other degenerated fibers in the left temporo-pontine tract appeared to have been related to the cortex. The right temporo-pontine tract exhibited much less degeneration, only its more ventral fibers having been injured at the base of the putamen, with a few fibers of the right temporo-pulvinar fasciculus (fig. 4). On the right side the pulvinar showed black granules in its medial and caudal part (fig. 2); on the left, these granules were less in number. This area of the pulvinar in which the black granules were seen (fig. 2) could be compared quite well to a similar area shown in figure 25 (section 151) of Walker ('38). In the part of the pulvinar illustrated in this figure no retrograde degeneration was visible, although the animal had suffered a partial hemidecortication (experiment 6, page 109) which spared the temporal lobe. In the present material the lesion 
involved chiefly that area which was left intact in Walker's case 6 . The black granules appear to represent cellular changes following the interruption of thalamo-cortical fibers which have their origin in this particular area of the pulvinar (dorsomedial part). On the basis of the lesions in this case and those to follow, one would judge that these fibers end along the hippocampal or inferior temporal gyri, behind the level of the amygdala. Both medial geniculate nuclei showed many dark granules, apparently due to involvement of the superior temporal gyri, which were injured in about the same area on both sides. The right medial geniculate nucleus is illustrated in figure 2. Other degenerated fibers were found in the capsulae externa and extrema of both sides, in the anterior commissure, and in the corpus callosum. A few degenerated fibers were present in the optic radiations and in the ventral components of both divisions of the left internal cortico-tectal tract (Crosby and Henderson, '48).

Bucy and Klüver ('40) carried out an extensive bilateral extirpation of the temporal lobes of a macaque. After a suitable period the animal was killed and the brain prepared by the Weil technique. Their lesions included most of the amygdala and the greater part of the hippocampus. Among the paths degenerated were the temporo-frontal tracts, association fibers to undestroyed portions of the parietal, occipital, and posterior frontal regions of the cortex, the anterior commissure, except its interbulbar component, the temporo-pulvinar tracts, about three-fifths of each fornix, a small part of the visual radiations on the right and more on the left and part of each stria terminalis.

There were evidences of retrograde degeneration in the ventrolateral portion of the left lateral geniculate and in the posterior tips of the medial geniculate nuclei, with the more extensive degeneration on the right. Certain other areas, as the putamen and the tail of the caudate nucleus, were likewise involved in the lesion. For further details the original paper should be consulted. It is obvious that 
many of the tracts reported as containing degenerated fibers in the present paper are comparable to those wholly or partially degenerated by Bucy and Klüver. Such differences as do exist are due largely to differences in extent, depth, and precise location of the lesions and to the fact that Bucy and Klüver employed the Weil and the present author the Marchi technique.

Summary. This monkey, which had learned to discriminate perfectly between the form and the weight of various objects and to recognize the objects, did not present any changes in ability for discrimination or in behavior following a left-sided lesion which involved a little surface cortex of the temporal lobe, the lateral olfactory tract, the prepiriform cortex, and only the dorsal part of the amygdala and which destroyed the base of the claustrum and of the capsula externa and capsula extrema and also a few fibers of fasciculus uncinatus. Farther caudally the lesion destroyed much of the temporopontine fibers and partially involved the putamen, the temporo-pulvinar fasciculus, and the hippocampus. A second lesion on the right destroyed completely the amygdala and the uncus, the rostral end of the hippocampus, and the hippocampal and inferior temporal gyri, in addition to a little surface cortex. Following this second lesion the monkey could no longer discriminate between the form of objects and he showed a greater tameness. The animal presented this same picture after destruction of part of the superficial cortex of the temporal lobe on the left side. Following removal of more cortex of the temporal lobe on the right side, the animal could neither discriminate between the form of the boxes nor recognize various objects placed on a board, although still able to appreciate differences in weight. The tameness had increased. All objects which were presented had to be smelled and licked before the animal ate them, if they were edible, indicating that it could no longer discriminate between them by sight alone but had to use also the senses of smell and of taste. 


\section{Monkey no. 4}

This monkey was tested for the recognition of various objects and the ability to discriminate between them by their form before it was operated on May 27, 1949. Through a bilateral opening in the skull over area 6 , needles were introduced into the brain substance with the help of the stereotaxic apparatus, the aim of this operation being to destroy the columns of the fornix. No changes in behavior and no loss of discrimination followed this first bilaterally placed lesion.

A week later (June 3,1949 ) the external and extreme capsules were destroyed on either side with the help of the stereotaxic instrument. No changes in behavior were recorded afterward and all previously learned tests were perfectly recalled; moreover, the monkey learned quickly to discriminate between differences in weight (see Material and Technique). The animal was reoperated on June 14, 1949, and the rostral half of the left temporal cortex was removed. Postoperative testing disclosed no loss of discrimination and the behavior of the animal seemed normal. Ten days later (June 24, 1949) a lesion was made similar to the last one, but on the right side. Again no changes in behavior and no loss of discriminatory ability were recorded.

On July 1, 1949, a lesion was placed in the left hippocampal gyrus and the surrounding structures. Upon manipulation of the instrument in the medial and ventral region of the temporal pole (apparently the hippocampal gyrus) changes in respiration were noted, consisting of an arrest of respiration followed by a deep inspiration. In spite of all these lesions, the animal showed no loss of discriminatory ability when the usual tests were made and appeared normal in behavior.

Finally, on July 8, 1949, the right hippocampal gyrus was destroyed and the same changes in respiration were noted. Only after this last lesion was some loss in discriminative power observed. The animal was tested for the discrimination 
of weight (using $50 \mathrm{gm}$ and $200 \mathrm{gm}$ ) and this was recalled perfectly. When retested for discrimination between objects by their form, the monkey appeared to have forgotten completely what it had previously learned in order to obtain food. It pulled in either box indiscriminately. However, after a few days, it succeeded in selecting the proper box, but only after a greater number of trials than had been required to learn the difference the first time. Furthermore, instead of pulling in the correct box directly as before, the animal had to shift at each trial; only after repeated trials did it pull in the correct box. These results suggested that it had to use other cues to supplement its inability to discriminate by sight. To eliminate all possible cues other than visual, the boxes were reweighed and found to be of the same weight and paper was glued under each box to equalize the sound. On repetition of the test, the correct box was pulled in directly each time. Then the paper floors were removed, but there was no change in the results. Finally, with only a few errors, the monkey could discriminate between one box and the other by the noise produced by the rubbing of the boxes on the floor. This result suggested that the animal had learned to discriminate between the boxes by using sound when, after the last operation, it was shifting at each trial.

The animal was tested for the ability to discriminate between white and gray. Only after repeated trials (more than usual) could the monkey differentiate one box from the other. However, discrimination by sound - using a bell connected with one of the two boxes - was learned much more quickly. Autopsy of this animal was done on July 28, 1949.

Macroscopic and microscopic anatomy. Each lesion could be individualized and most of the degenerated tracts could be traced from a definite lesion and thus a correlation between function and structure was possible. The first two lesions were made the same day and as symmetrically as possible, with the help of the stereotaxic instrument. On the right side the 
structures involved in the lesion were the following: a little surface cortex of area 6 , the cingulum, the corpus callosum, the fornix, the stria medullaris, and the mammillo-thalamic tract. On the left side the following structures were involved: a little surface cortex of area 6 , the corpus callosum, the head of caudate nucleus, the left stria medullaris, the mammillothalamic tract, and the anterior limb of the internal capsule, in addition to a few fibers of the ansa lenticularis.

The corpus callosum showed many degenerated fibers (fig. 5) due to lesions on the surface cortex and in the commissure itself. The degenerated fibers in the right cingulum were numerous rostral to the lesion (fig. 5), decreasing in number as they were followed anteriorly; behind the lesion, fewer fibers showed Marchi granules (fig. 6). The degeneration in the right fornix was in the lateral third of this structure (fig. 6); these degenerated fibers could be traced chiefly to the corresponding lateral septal nucleus. Both striae medullares were largely degenerated, only the more medial fibers of the left stria medullaris being intact (fig. 7). These fibers were destroyed at the point where the various components of the stria medullaris join each other. The degenerated fibers could be traced to the habenulae and were seen decussating in the habenular commissure (fig. 8). Both mammillo-thalamic tracts showed degenerated fibers which could be traced to the anterior thalamic nuclei, these fibers being more numerous on the left side (fig. 6). The anterior limb of the left internal capsule was destroyed in about its lower third for a considerable extent. As a consequence, many degenerated fibers could be traced within the left fronto-pontine tract and the interconnections between the thalamus and the frontal cortex showed degeneration on the left side; most of these fibers come from or go to the left dorsomedial nucleus of the thalamus (fig. 7). The second set of lesions were caudal and lateral to the previous destructions. On both sides the needle of the stereotaxic machine went through the cortex, the corona 
radiata, the external capsule, and the claustrum. The lesion was placed so that the capsula externa and the claustrum were destroyed together with a few fibers interconnecting the temporal gyri,

The next two lesions, made 10 days apart, involved the cortex on the lateral side of both temporal lobes for about their anterior half. The superior and middle temporal gyri were destroyed rostrally and, in addition, a little cortex at the base of the frontal lobe. From these two lesions there resulted a degeneration of the fibers interconnecting the superior, middle, and inferior temporal gyri. Degenerated fibers, probably due to the lesions, were present in both temporopontine tracts.

The last two lesions, one made a week later than the other, involved the under surface of the temporal lobe, partially destroying, on either side, the ventral part of the inferior temporal gyrus and the hippocampal gyrus. Some of the degenerated fibers leaving the inferior temporal gyrus entered the transverse limb of the anterior commissure on either side. Farther caudally, two major groups of degenerated fibers accumulated ventral to the anterior commissure; the more dorsal of these fibers could be traced in large number into the temporo-pontine tract. Some of the more ventral fibers accumulated laterodorsal to the tail of the caudate nucleus; then, probably as temporo-pulvinar fascicles, they could be traced to the caudal and lateral part of the pulvinar. At first these fibers lay laterodorsal to the tail of caudate nucleus, but became mediodorsal to this structure as they ran caudalward, being separated from the lateral geniculate nucleus by the external cortico-tectal tract which appeared intact in this brain. Other degenerated fibers passed caudalward through the pulvinar (fig. 9) to the superior colliculus on either side; these fibers, which belong to the ventral components of the internal cortico-tectal tracts, presumably had their origin in the area destroyed by the more caudal part of these two last lesions which involved the inferior and the 
hippocampal gyri far caudally in the occipital lobe. The dorsomedial portion of the candal part of each pulvinar (fig. 9) showed a strong concentration of black granules which probably represented cellular disintegration resulting from the destruction of the surface cortex along the basal part of the temporal lobe.

From the lateral and rostral area of the left amygdala, which was partially injured by the instrument, fibers were traced into the lateral component of the left stria terminalis. These fibers, swinging forward with the caudate nucleus, occupied a ventromedial position in the stria terminalis (Lauer, '45). They could be traced only to the left stria medullaris, which was injured by a previous lesion.

Summary. After lesions which involved the striae medul. lares, the mammillo-thalamic tracts, the capsula externa and capsula extrema partially, and most of the interconnections of the left dorsomedial thalamic nucleus with the frontal cortex, in addition to a few fibers of the right fornix and right cingulum, no appreciable personality changes and no loss of discrimination were observed in this animal. Destruction of other areas in the rostral half of each temporal lobe produced no observed changes in behavior. Retesting for discrimination disclosed no loss of memory for previously learned skills.

Only after bilateral destruction of the inferior temporal and hippocampal gyri was addẹd to the previous lesions did the animal show a lack of visual discrimination; however, with the aid of other cues, like sound, it was able to relearn to discriminate between the shape of objects but it took him many more trials than previously. To learn a new test based on visual cues, more than the normal number of trials were required. Tests based on auditory cues were learned very rapidly.

Apart from this temporary loss of memory for visual form discrimination and a slowness in relearning or learning for the first time tests based on visual cues, this monkey showed no changes in behavior. 


\section{Monkey no. 5}

This monkey was tested for the ability to discriminate between the form, the sound, and the weight of objects. It performed the last 50 trials of the test for form without error, pulling in directly the box which contained food. It also learned to discriminate perfectly between differences in sound and between differences in weight, testing at each trial before pulling in the correct box. Following these preliminary tests, lesions were placed (July 29, 1949) in the region of the amygdala on either side of the brain. Upon manipulation of the instrument on the deeply situated structures, changes in respiration, similar to those obtained during the operation on monkey no. 4 , were observed.

It was surprising, a few hours after the operation when the effect of the ether had surely disappeared, to find the animal apparently sleeping, in a state comparable to that of the monkeys who were asleep following hypothalamic lesions (Ranson, '39). At first the animal lay on its side, with eyes half closed. Loud noises or intense light brought only a weak response, the animal opening its eyes and moving its head slightly. It would not eat by itself, so feeding was done intravenously, using a solution of amino acids and dextrose. Rectal temperature taken on the first day indicated $99.7^{\circ} \mathrm{F}$. (6 P.м.); respiratory rate was 30 and heart rate 85 . On the second day after the operation, the animal sat most of the time with head bent forward and eyelids drooping (fig. 10). Intravenous injection was repeated. When the animal was handled, it responded by jerky movements of the limbs. Rectal temperature remained at $99.7^{\circ} \mathrm{F}$. ( 11 A.M.). On the third day its attitude remained unchanged; it was drowsy most of the time. Intravenous injection was repeated. However, its rectal temperature indicated a rise toward normal, reaching $98.2^{\circ} \mathrm{F}$. at 10 A.M. and $100.3^{\circ} \mathrm{F}$. at 4 P.M.

On the next day (4th), the animal began to awaken; it responded to less intense stimuli than on the first day after the operation. At the end of the day it was taking any object 
which was handed to it, smelling and eating the object, if edible. For instance, the monkey ate pieces of banana, orange, and apple, and a few raisins, but discarded, after smelling them, pieces of bread and casein cubes which were familiar to it prior to the operation. Pieces of bread covered with white sugar, of which monkeys are usually very fond, were presented to the animal; it smelled them and discarded them. Although it could feed itself, it still had a strong tendency toward the sleeping posture; for instance, it was seen a few times in that typical posture with a piece of banana held to its lips. On a few occasions it picked up various objects directly with its mouth, using its hands only to hold them to its nose.

Beginning with the 5th day after operation the animal gradually became active until by the 12 th day it had resumed almost normal activity, eating the usual food, including bread and casein. Even 11 days after the operation it could still be seen sitting with head bent forward, especially if the room were quiet. As the animal became more active a right hemianopsia could be observed, evidenced by the fact that it picked up only the objects which were placed in its left visual field and it circled around to the left when it wished to reach to the right. In addition, observation disclosed a little weakness of its left limbs which disappeared in a few days. It remained very tame and very easy to handle.

Twelve days after the operation the animal was considered normal enough to repeat the discrimination tests. It was retested first for the discrimination of form. On the first few trials it made many errors, showing a strong tendency to pull the box on the left, as was to be expected with a right hemianopsia. In spite of this visual defect, the animal managed to relearn the visual test in half as many trials as preoperatively. For instance, it made 21 errors in the first 30 trials, showing a strong tendency to the left; of all the errors made in the following 40 trials, however, only two errors were made in the last 25. It took the monkey only a few trials to carry out the test for weight discrimination as well as pre- 
operatively. After 40 trials the animal chose every time the lighter of the two boxes to obtain food. Sound discrimination was almost perfectly recalled, the monkey making only a few errors in the first few attempts. It was sacrificed on August 15, 1949, and perfused with $10 \%$ formalin.

Macroscopic and microscopic anatomy. On the right side the lesion involved the superior temporal gyrus through which the instrument was introduced; the gyrus was destroyed along the middle third of its caudo-rostral extent. Deeper, there was a slight involvement of the insular cortex. Rostrally the lesion included most of the amygdala, only the dorsal part of this structure being spared (fig. 11). The anterior half of the hippocampal gyrus, or its connections, and, caudal to the amygdala, the rostral two-thirds of the hippocampus, together with the fimbria, were destroyed. Laterally, the transverse limb of the anterior commissure and the basal part of the capsula extrema were partially severed. Dorsal to the hippocampus the tail of the caudate and a few fibers of the temporo-pontine and temporo-pulvinar tracts and of the optic radiations were injured in the ventrolateral part of the putamen. The caudal part of the lesion involved chiefly the hippocampus and the hippocampal gyrus.

On the left side the instrument was introduced through the superior temporal gyrus, which was destroyed in about the middle third of its extent; deeper the fibers interconnecting the superior and middle temporal gyri were interrupted. Rostromedially (fig. 12) the uncus and the amygdala were completely destroyed. Rostrolaterally (fig. 12) the instrument severed the fasciculus uncinatus, the base of the capsula extrema and of the claustrum, the temporal limb of the anterior commissure, and, a little more caudally, the transverse limb of this same commissure (see Bucy and Klüver, '40). Behind the anterior commissure the lesion extended dorsally to the base of the putamen and of the globus pallidus, involving also the ventral part of the ansa lenticularis and, below this structure, fibers of the supraoptic system. Behind the amygdala only the very rostral end of the hippocampus 
was destroyed, but the fimbria was injured in about the anterior half of its extent (fig. 13). The rostral part of the hippocampal gyrus was likewise injured and farther back its interconnections were severed for quite a distance. As the lesion was followed caudally, it shifted more dorsally; still farther back it extended more medially as well. Above the hippocampus the tail of the caudate nucleus and the stria terminalis were transected (fig. 13); dorsal to these structures the globus pallidus and the putamen were destroyed and, with this last structure, fibers belonging to the temporopontine and temporo-pulvinar tracts (fig. 13). Medial to the putamen the optic tract was severed just before its entrance into the lateral geniculate nucleus (fig. 13). As the lesion was traced still farther back (fig. 14) structures showing destruction could be seen in the following order: the putamen, the posterior limb of the internal capsule, the lateral nucleus of the dorsal thalamus, and, finally, at the caudal end of the lesion, the posterior commissure and the commissure of the superior colliculus, all on the left side.

On the left side, stria terminalis was transected completely and degeneration could be seen in the fibers of its two components, the medial and the lateral. The degenerated fibers of the lateral component could be traced to the corresponding habenula; the fibers in the medial component distributed largely to the bed nuclei of stria terminalis and of the anterior commissure on the same side (fig. 15). On the right side, corresponding to an involvement of the ventral part of the amygdala, stria terminalis showed degeneration chiefly in the fibers of its lateral component, these passing to the left habenula; only a few fibers of the medial component showed degeneration and these could be traced chiefly to the bed nucleus of the right stria terminalis (fig. 15). Degenerated fibers were also seen bilaterally in the fornix; some of these fibers ended in the lateral septal nucleus (fig. 15), others ran in the postcommissural fornix toward the habenula and toward the hypothalamic area. Those fornix fibers ending in the habenula appeared to terminate more laterally than those derived 
from the stria terminalis. Other degenerated fibers, the origin of which could not be determined, were traced into the preoptic and the hypothalamic areas. They formed, on either side, a group of fine fibers which ran ventrally close to the ventricle. At the level of the mammillary body some of the fibers decussated to the opposite side and were seen dorsolateral to this structure; from this point they became more ventral as the mammillary body disappeared and they continued their course behind this nucleus into the basal part of the midbrain where they disappeared.

Conspicuous degeneration, especially on the left side, could be seen along fibers coming from the piriform area and could be traced to the rostral area of the lateral septal nucleus. Other degeneration resulting from the rostral part of the lesion was found in the anterior commissure and in the left capsula extrema. A little more caudally, degenerated fibers were seen in the supraoptic system and in the left ansa lenticularis, fasciculus lenticularis, and optic radiations. Farther back the temporo-pulvinar fasciculi showed fine degenerated fibers and large degenerated fibers were present in both temporo-pontine tracts but were more numerous on the left. The posterior limb of the left internal capsule also showed some degeneration.

Finally, the more caudal structures showing some degeneration, all on the left side, were as follows: the external and internal cortico-tectal tracts, the superior collicular and posterior commissures, the medial tecto-spinal tract, and fibers of the mesencephalic root of $V$ (fig. 16). These results should be compared with those of Bucy and Klüver ('40) reviewed on page 218 .

Summary. This animal learned preoperatively to discriminate between differences in shape, weight, and sound. Lesions were then placed in the temporal poles. Some of the structures involved bilaterally were as follows: the rostral part of the hippocampus and of the hippocampal gyrus or their interconnections, the anterior commissure, the temporo-pulvinar and temporo-pontine fibers, and a little surface cortex of the 
superior temporal gyrus. Other structures injured more on the left side were the amygdala, the putamen, the claustrum, and the capsula extrema. Finally, the globus pallidus, the ansa lenticularis, the lateral thalamic nucleus, the optic tract, and the internal capsule were destroyed in part on the left side, but not affected on the right side. From these structures degeneration was traced into the several tracts and nuclei.

Following these lesions, the animal was in a state resembling sleep; for the first three days it sat with head bent forward and eyes half closed. During that time it had to be fed intravenously and its temperature was subnormal. On the 4th day following the operation, it gradually became more active until on the 12th day it appeared normal in behavior except for a greater than usual tameness which persisted. When this animal began to come out of its drowsiness, it ate only the food it could identify by smell, discarding other food, even that of which it was very fond (as for instance white sugar). After a few days the dietary habits became normal.

Retesting was possible 12 days after the operation and no marked loss of discrimination was observed. The animal relearned quickly to discriminate between differences in form in spite of a complete right hemianopsia. Weight discrimination was also relearned after a few trials only. When the ability to discriminate between sounds was tested, it was found that the animal could repeat the test as well after the operation as before, except for a few errors at the first attempts.

\section{Monkey no. 6}

No tests were carried out on this animal, for the chief interest in the case was the investigation of some of the anatomical structures, the destruction of which could cause a sleeplike state. On September 17, 1949, the skull was trepanned and lesions were placed bilaterally in the caudal part of the hippocampus through an opening in the temporal cortex. Using the same bilateral routes, on October 6, 1949, the 
rostral area of the temporal lobe was injured along its basal surface. During this last operation, changes in respiration similar to those reported for cases 4 and 5 were observed upon contacting the medial and basal portions of the temporal poles. Following these two bilateral lesions, no changes in behavior were observed, the animal being as wild as ever. Autopsy was done on October 25, 1949.

Macroscopic and microscopic anatomy. Postmortem study of this brain revealed that, on the left side, the lesion extended rostrocaudally along the ventrolateral surface of the temporal lobe. Rostrally the cortex was injured where the middle and the inferior temporal gyri meet on the basal surface; caudalward the lesion extended more laterally and involved the lateral part om the inferior temporal gyrus and, to a lesser extent, the middle temporal gyrus; in the caudal half the injury was entirely along the basal surface of the inferior temporal gyrus and was deeper. In this caudal part of the lesion, the interconnections of the hippocampal and inferior temporal gyri were severed along the ventrolateral part of the alveus. In other words, the lesion extended along areas 20 and 19 rostrocaudally and encroached upon area 21 rostrally.

The superior and the middle temporal gyri were partially injured, more rostrally on the right side than on the left. Deeper on the right, the rostral end of the lesion involved the caudal part of the amygdala and the underlying hippocampal gyrus. Caudal to the amygdala the rostral part of the hippocampus was completely destroyed, but still farther back more and more of the dorsal and medial parts of this structure were spared. At the caudal end, only the basolateral portions of the cornu Ammonis and of the gyrus dentatus were injured. Lateral to the hippocampus, the interconnections of the hippocampal and the inferior temporal gyri were cut, together with some fibers of the optic loop.

As a result of these superficial and deep lesions a few tracts showed degenerated fibers. (See also review of paper by Bucy and Klüver, '40, on page 218.) The anterior com- 
missure presented many degenerated fibers from the left lesion; the temporo-pulvinar, temporo-pontine, and external cortico-tectal tracts were largely destroyed on the right, and, to a lesser extent, on the left. Cortico-geniculate fibers showing degeneration were present only on the right side. The fornix was greatly degenerated on the right but very little on the left (fig. 17). The internal cortico-tectal tracts of both sides showed many degenerated fibers in the ventral components of both the preoccipital and occipital divisions. Finally, the right stria terminalis exhibited a few fibers containing Marchi granules.

Summary. This animal was operated upon twice; a first lesions was placed in the caudal and basal part of the temporal lobe along areas 20 and 19 bilaterally, and a second lesion involved the rostral part of the temporal lobe, also on both sides. In addition, the lateral temporal cortex was partially injured along the superior and middle temporal gyri on the right side and along the middle and inferior temporal gyri on the left side. No changes in behavior were observed following these two sets of lesions.

Apart from the cortex, the main structures destroyed were, on the right side, the caudal part of the amygdala, most of the hippocampus, and certain fascicles from the middle and inferior temporal and the hippocampal gyri caudal to the amygdala; and, on the left, fibers from the inferior temporal and the hippocampal gyri at the level of area 19.

\section{DISCUSSION}

In this series only one animal (monkey no. 2) showed impairment in weight discrimination; this test, however, which the animal could not learn after repeated trials, was introduced postoperatively. Ruch, Fulton and German ('38) observed that, in monkeys, discrimination of lifted weight was partially and permanently lost only after a lesion which involved the posterior parietal lobule together with the postcentral gyrus. In the present material very little of the lower part of the parietal cortex was destroyed, but the insula and 
many of the interconnections of the parietal cortex were injured on both sides. Since the test was introduced only after bilateral lesions and since similar findings have not been reported in the literature consulted, the author can draw no definite conclusions on the basis of this single result. Two animals presented an impairment of form discrimination. Case 3 showed a complete loss, which was never overcome; case 4 relearned the visual test only after repeated trials and apparently with the help of other cues. Several lesions were placed in the brains of these two animals. However, this loss of visual discrimination appeared only after destruction along the basal surface of the temporal lobe on either side. In monkeys nos. 2 and 5 no loss of discrimination was observed following lesions on the basal surface of the brain, but in these animals the brain lesions did not extend so far caudally as in monkeys nos. 3 and 4 , where more of the visual association cortex and of its interconnections were injured. Ades and Raab ('49) observed permanent loss of form discrimination only when a bitemporal decortication was added to removal of areas 18 and 19. In monkey no. 3 a permanent loss of the ability to discriminate by form was obtained following lesions which destroyed the basal cortex and its interconnecting fibers along the temporo-occipital surface of the brain but which involved very little of the temporal cortex on the lateral surface of the brain. However, after decortication of the caudal area of both temporal lobes had been added to the previous lesions, the monkey showed a greater loss of visual discrimination. The animal could no longer discriminate by sight alone and resembled, in this respect, the monkeys studied by Klüver and Bucy ('39) which showed psychic blindness. The production of this visual agnosia appears to depend upon the bilateral destruction of area 19, and possibly also of area 18 , in addition to that of the caudal and basal area of the temporal lobe, which is much more under the influence of the visual association cortex than its more rostral and dorsal part (Bailey et al., '43, and '44; 
see also McCulloch's diagram, fig. 39, p. 81, in Bonin and Bailey, '47).

The monkeys were tested for the ability to discriminate by sound following variously placed lesions in the temporal lobes. In all of these lesions, the auditory association cortex was only partially destroyed. The sense of smell appeared to be intact in all cases, even after extensive lesions which included the amygdala, the piriform lobe, and the hippocampus. These results are in agreement with the findings of Swann ('34) in the rat and with those of Allen ('41) in the dog.

None of the writer's monkeys showed the complete polysymptomatic picture described by Klüver and Bucy ('37, '38, '39) following bilateral temporal lobectomy. However, one animal referred to earlier in this discussion presented a visual agnosia which can be compared to the psychic blindness described by these two observers. It appears to the present author that, although the other symptoms of the bitemporal monkey may depend for their production upon complete and bilateral destruction of the temporal lobes, this particular symptom of psychic blindness can be observed following lesions which are restricted on both sides to the caudal and basal area of the temporal lobe and which include area 19, together with (or without) area 18.

Another alteration of normal behavior observed in 4 of the animals studied was a change toward greater tameness, following lesions which involved mainly the rostral area of the temporal lobes including the olfactory formations on the mediobasal surface. Other symptoms, such as oral tendency, hypermetamorphosis, and hypersexuality, which were observed by Klüver and Bucy, were not seen in the monkeys of this series following differently placed destructions in the temporal lobes. Probably these symptoms failed to appear because the lesions were not sufficiently extensive, as Klüver and Bucy ('39) reported after various subtotal destructions in the temporal lobes. 
Finally, an unexpected result was obtained in monkey no. 5 that showed a state resembling sleep following bilateral lesions in the temporal lobe. The chief structures bilaterally destroyed were the amygdala, the piriform cortex, and the hippocampus. The hypothalamus was entirely spared. That some of the structures thus destroyed exert an influence on the hypothalamus or midbrain appears to the writer the more plausible explanation of this single result; for degenerated fibers, the origin of which, however, cannot be determined exactly in the material available, were found on either side of the hypothalamus, and some of them were seen to bypass the mammillary bodies to reach the midbrain. In addition to somnolence, hypothermia was observed in monkey no. 5 and respiratory changes were noted when the medial side of the temporal pole was contacted, an effect in agreement with the findings of Kaada, Pribram, and Epstein ('49). The results suggest that the amygdaloid area is one of the brain centers concerned with keeping the animal awake. Bilateral subtotal hippocampal destruction did not put him to sleep.

While this paper was being prepared for publication, another animal (fig. 19) showed a sleep-like state similar to that of monkey no. 5, following bilateral lesions placed with the stereotaxic instrument in the hippocampal formation and amygdaloid area (December 27, 1950). Microscopic study of the brain of this case will be reported later.

\section{SUMMARY}

This report is concerned with anatomical and experimental studies of the brains of 6 monkeys in which various lesions had been placed. All of these involved the temporal lobes. Five of these animals were tested pre- and postoperatively for form discrimination, and some of them for weight and sound discrimination.

Sound discrimination was preserved in all cases. One animal could not learn to discriminate differences in weight postoperatively, following brain lesions which destroyed, on both sides, a large part of the insula and many interconnec- 
tions from the parietal cortex. Another animal had forgotten completely form discrimination following extensive bilateral injury to the fibers of the visual association cortex. Another monkey could relearn to discriminate form after a lesion similar to, but less extensive than, that suffered by the previous animal.

Respiratory changes were observed upon touching the temporal pole medially. One monkey (case no. 5) was asleep, and then drowsy, after a bilateral destruction involving chiefly the medial and basal portions of the temporal pole, perhaps most significantly the amygdaloid area.

Finally another animal (not belonging to the same series) showed a state resembling sleep following bilateral destruction in the basomedial area of the temporal lobe.

\section{LITERATURE CITED}

ADES, H., AND D. RAAB 1949 Effect of preocipital and temporal decortication on a learned visual discrimination in monkeys. J. Neurophysiol., 12: 101-108.

ALLEN, W. F. 1941 Effect of ablating the pyriform-amygdaloid areas and hippocampi on positive and negative olfactory conditioned reflexes and on conditioned olfactory differentiation. Am. J. Physiol, 132: 81-92.

Bailey, P., G. v. Bonin, E. W. Davis, H. W. Garol and W. S. MoCulloch 1944 Further observations on associational pathways in the brain of Macaca mulatta. J. Neuropath, and Exp. Neur., 3: 413-415.

Bailey, P., G. v. Bonin, H. W. Garol AND W. S. McCulioch 1943 Funetional organization of temporal lobe of monkey (Macaca mulatta) and chimpanzee (Pan satyrus). J. Neurophysiol, 6: 121-128.

BonIN, G. V., AND P. BAILEY 1947 The neocortex of Macaca mulatta. The University of Illinois Press, Urbana, Illinois. $163 \mathrm{pp}$.

Bucy, P. C., AND H. KLüvfr 1940 Anatomic changes secondary to temporal lobectomy, Areh. Neur, and Psychiat., 44: 1142-1146.

Crosby, F. C., AND J. W. Henderson 1948 The mammalian midbrain and Isthmus regions. II. Fiber connections of the superior colliculus. B. Pathways concerned in automatic eye movements. J. Comp. Neur., 88: $53-92$.

DejpRtNe, J. 1895 Anatomie des centres nerveux. Rueff, Paris,2 vols.

KAada, B. R., K. H. Pribram AND J. A. Epstein 1949 Respiratory and vascular responses in monkeys from temporal pole, insula, orbital surface and cingulate gyrus. A preliminary report. J. Neurophysiol., 12: 347-356.

KL̈̈vfR, H. 1933 Behavior Mechanisms in Monkeys. Univ. of Chicago Press, $387 \mathrm{pp}$. 
KLÜver, H., AND P. C. Buor 1937 "Psychie blindness" and other symptoms following bilateral temporal lobectomy in Rhesus monkeys. Am. J. Physiol., 119: 352-353.

1938 An analysis of certain effects of bilateral temporal lobectomy in the rhesus monkey, with special reference to "psychic blindness.", J. Psychol., 5: 33-54.

1939 Preliminary analysis of functions of the temporal lobes in monkeys. Arch. Neur. and Psychiat., 42: 979-1000.

KRIEG, W. J. S. 1949 Connections of the cerebral cortex. II. The macaque. B. Material and methods. J. Comp. Neur., 91: 39-66.

LAUER, E. W. 1945 The nuclear pattern and fiber connections of certain basal telencephalic centers in the macaque. J. Comp. Neur., 82: 215-254.

RANSON, S. W. 1939 Somnolence caused by hypothalamic lesions in the monkey. Arch. Neur. and Psychiat., 41: 1-23.

Ruch, T. C., J. F. Fulton and W. J. German 1938 Sensory discrimination in monkey, chimpanzee, and man after lesions of the parietal lobe. Arch. Neur. and Psychiat., 39: 919-937.

Swank, R. L., AND H. A. Davenport 1935 Chlorate-osmic-formalin method for staining degenerating myelin. Stain Tech., 10: 87-90.

SWANN, H. G. 1934 The function of the brain in olfaction. II. The results of destruction of olfactory and other nervous structures upon the diserimination of odors. J. Comp. Neur., 59: 175-201.

WALKER, A. EARL 1938 The primate thalamus. Univ. of Chicago Press, pp. 109-112. 


\section{PLATES}

\section{ABBRFVIATIONS FOR PLATES}

AMYG., anygdala

ANT. COM., anterior commissure

ANT. COM. TEMP. L., anterior commissure, temporal limb

ANT. THAL. N., anterior thalamic nucleus BR. PONT., brachium pontis

CAUD. N., caudate nucleus

CING., cingulum

CLAUS., claustrum

CORP. CAL., corpus callosum

DOR. MED. THAL. N., dorsomedial thalamic nucleus

EXT. CAP., external capsule

EXT. CORT. TEC'I. TR., external corticoteetal tract

FIM., fimbria

FOR., fornix

FOR. BOD., body of fornix

FOR. POSTCOM., posteommissural fornix GL. PAL., globus pallidus

HAB. COM, habenular commissure

HIP., hippocampus

HIP. G., hippocampal gyrus

INF. TEMP. G., inferior temporal gyrus

INS., insula

INT. CAP., internal capsule
INT. CORT. TECT. TR., internal corticotectal tract

LAT. GEN. N., lateral geniculate nucleus

LAT. SEPT. N., lateral septal nucleus

LAT. THAL. N., lateral thalamic nucleus

LAT. VENT., Jateral ventricle

MAM. THAL. TR, mammillo-thalamie tract

MED. GEN. N., medial geniculate nucleus

MED. TECT. SP. TR., medial tecto-spinal tract

MES. R. V, mesencephalie root of $V$

MOT. N. $V$, motor nueleus of $V$

OPT. CH, optic chiasma

OPT. TR., optic tract

POST. COM., posterior commissure

PUL., pulvinar

PUT., putamen

ST. MED., stria medularis

ST. TERM., stria terminalis

SUP. COLL., superior colliculus

SUP. TEMP. G., superior temporal gyrus

TEMP. CORT. PONT. TR., temporo-corticopontine tract

TEMP. PUL, F., temporo-pulvinar fasciculus 
PLATES 1 TO 9

EXPLANATION OF FIGURES

1 Photomicrograph of a transverse section of the brain of monkey no. 2. This section which passes through pons shows degenerated fibers in the right temporo-pontine tract. Marchi preparation. $\times 11$.

2 to 4 Photomierographs of transverse sections of the brain of monkey no. 3. Figure 2 shows degeneration in the right pulvinar, the right medial geniculate nucleus, and the right stria terminalis and figure 3 in the rostral part of the lateral septal nuclei. $\times 11$. Figure 4 shows degeneration granules in the hippocampus, the hippocamal gyrus and other structures of the right side of the brain. Marchi preparations. $\times 7 \frac{1}{2}$.

5 to 9 Photomicrographs of transverse sections of the brain of monkey no. 4 . Figure 5 shows degeneration in the corpus eallosum and in the right cingulum; figure 6, more caudal, shows degeneration in the corpus callosum, the right eingulum, the striae medullares, the mammillo-thalamie tracts and the fornix. Figure 7 , still more eaudal, shows degeneration in the striae medullares and in the left dorsomedial thalamie nueleus. Degenerated fibers from the striae medullares and erossing in the habenular commissure are shown in figure 8 . Figure 9 shows degeneration in the left pulvinar and in the left medial geniculate nucleus. Marchi preparations. $\times 11$.

10 Photograph of monkey no. 5 three days after the operation.

11 to 16 Photomicrographs of transverse sections of the brain of monkey no. 5, prepared by Marchi method. Figure 11 shows the lesion on the right side. $\times 11$. Figure 12 shows the rostral part of the lesion placed on the left side. $\times 3$. Figures 13 and 14 show the lesion illustrated in figure 12, but at more caudal levels. $\times 7 \frac{1}{2}$. Figure 15 shows degeneration in the lateral septal nuelei and in the left stria terminalis. $\times 7 \frac{1}{2}$. Figure 16 shows degenerated fibers in the left mesencephalie root of $\mathrm{V}$ and in the left medial tectospinal tract. $\times 11$.

17 Photomicrograph of a transverse section of the brain of monkey no. 6, showing many degenerated fibers chiefly on the right side of the body of fornix. Marehi preparation. $\times 10$.

1.8 Photograph of the boxes used for visual form discrimination.

19 Plotograph of a monkey presenting a sleep-like state following bilateral lesions placed in the temporal pole. 

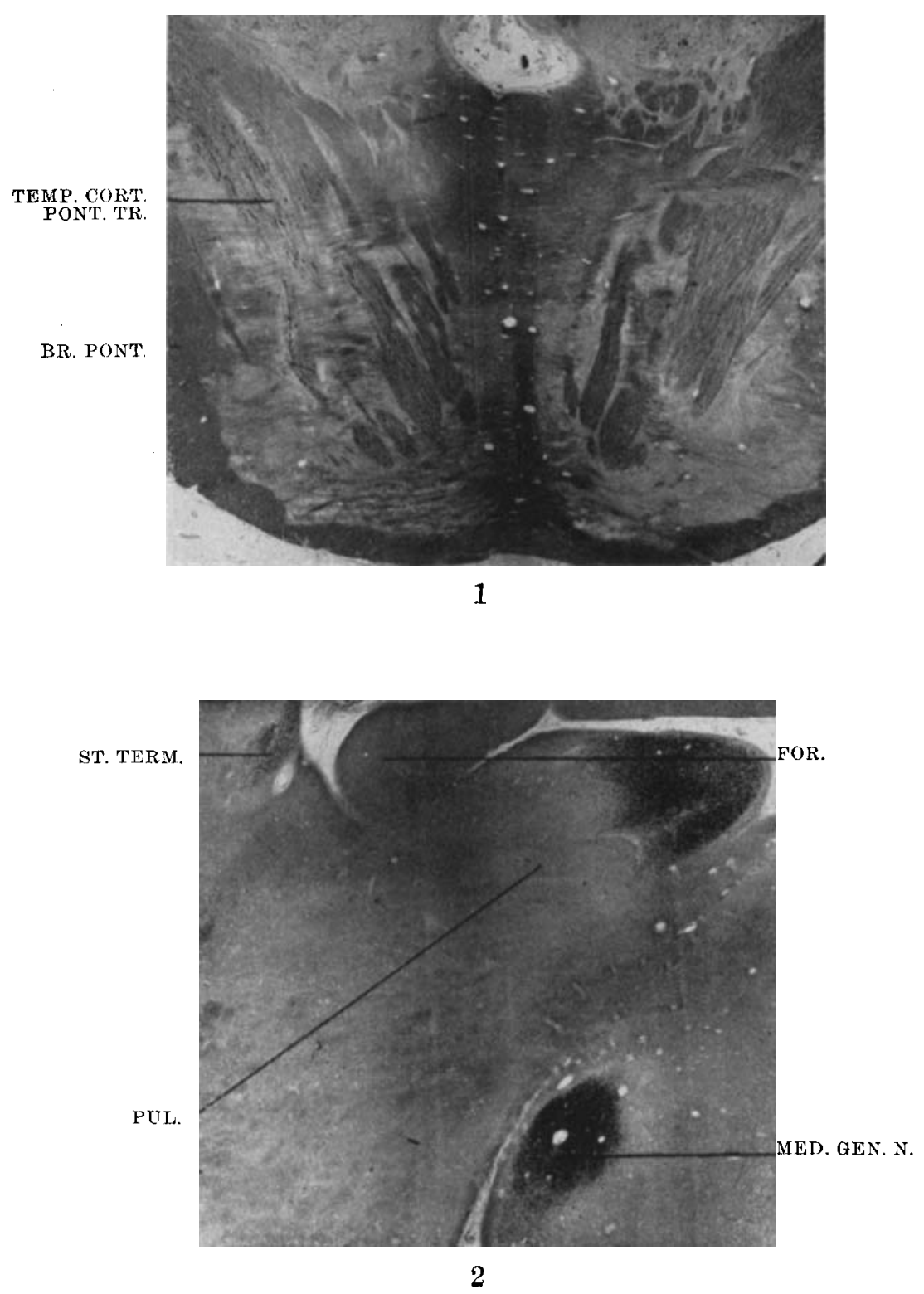

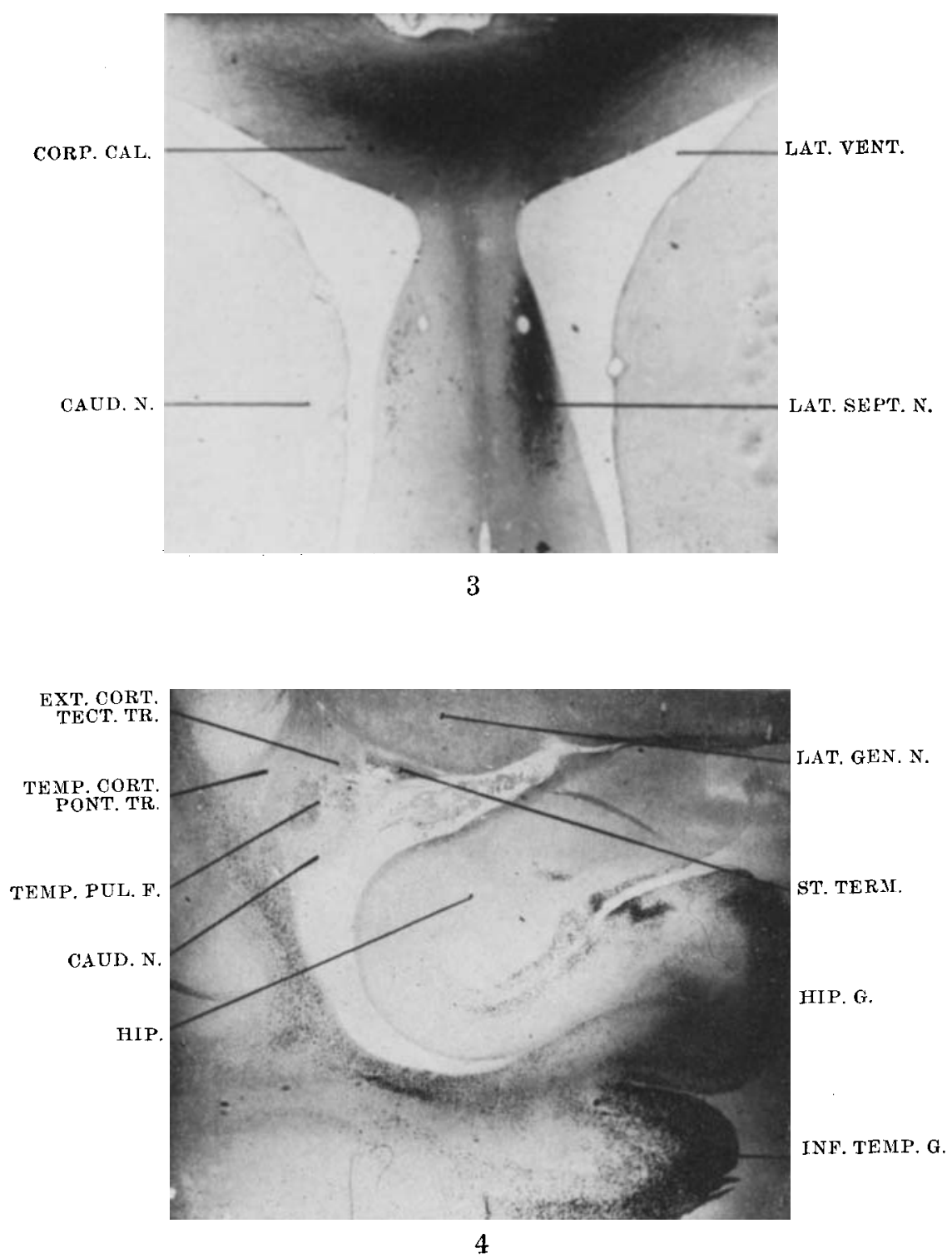

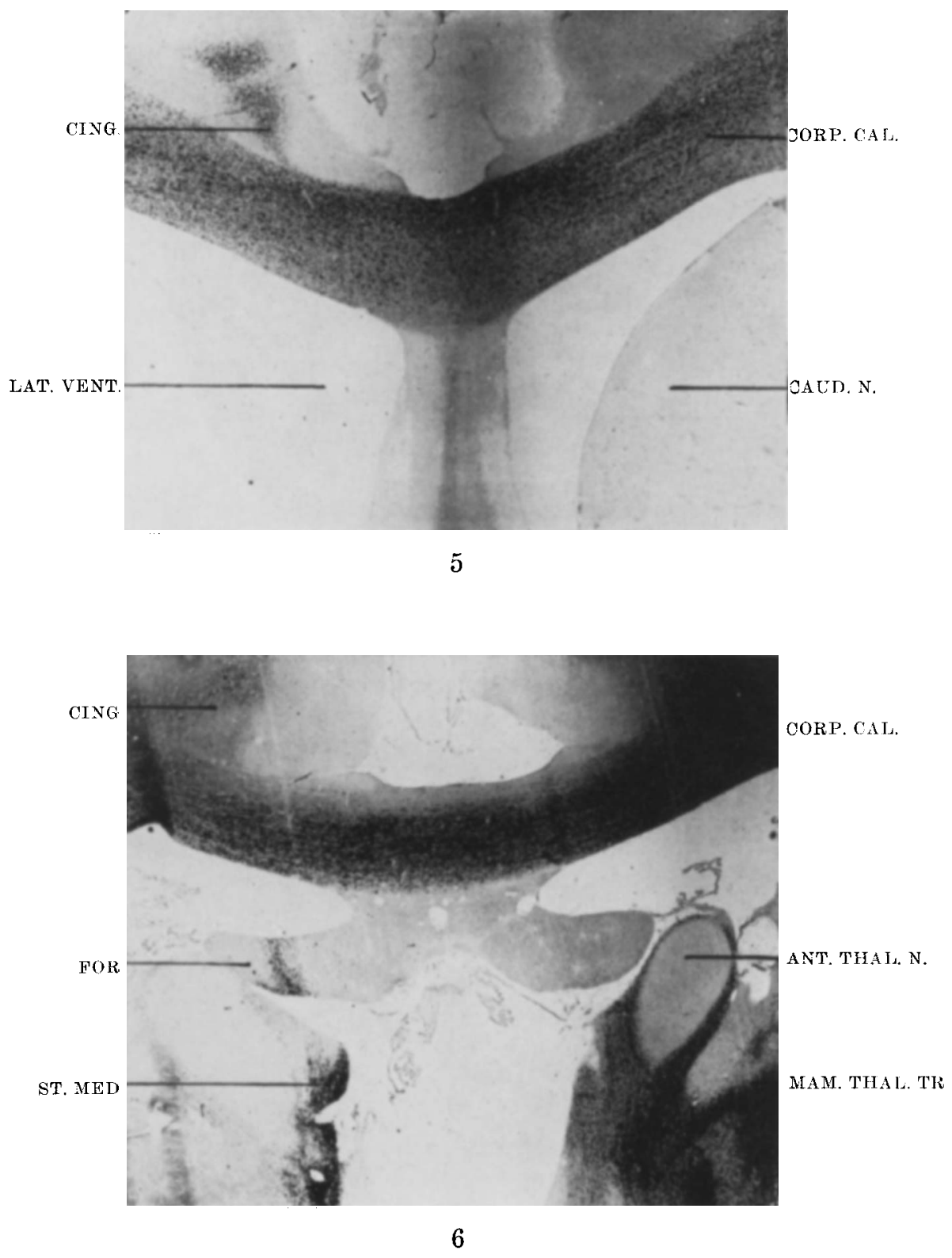

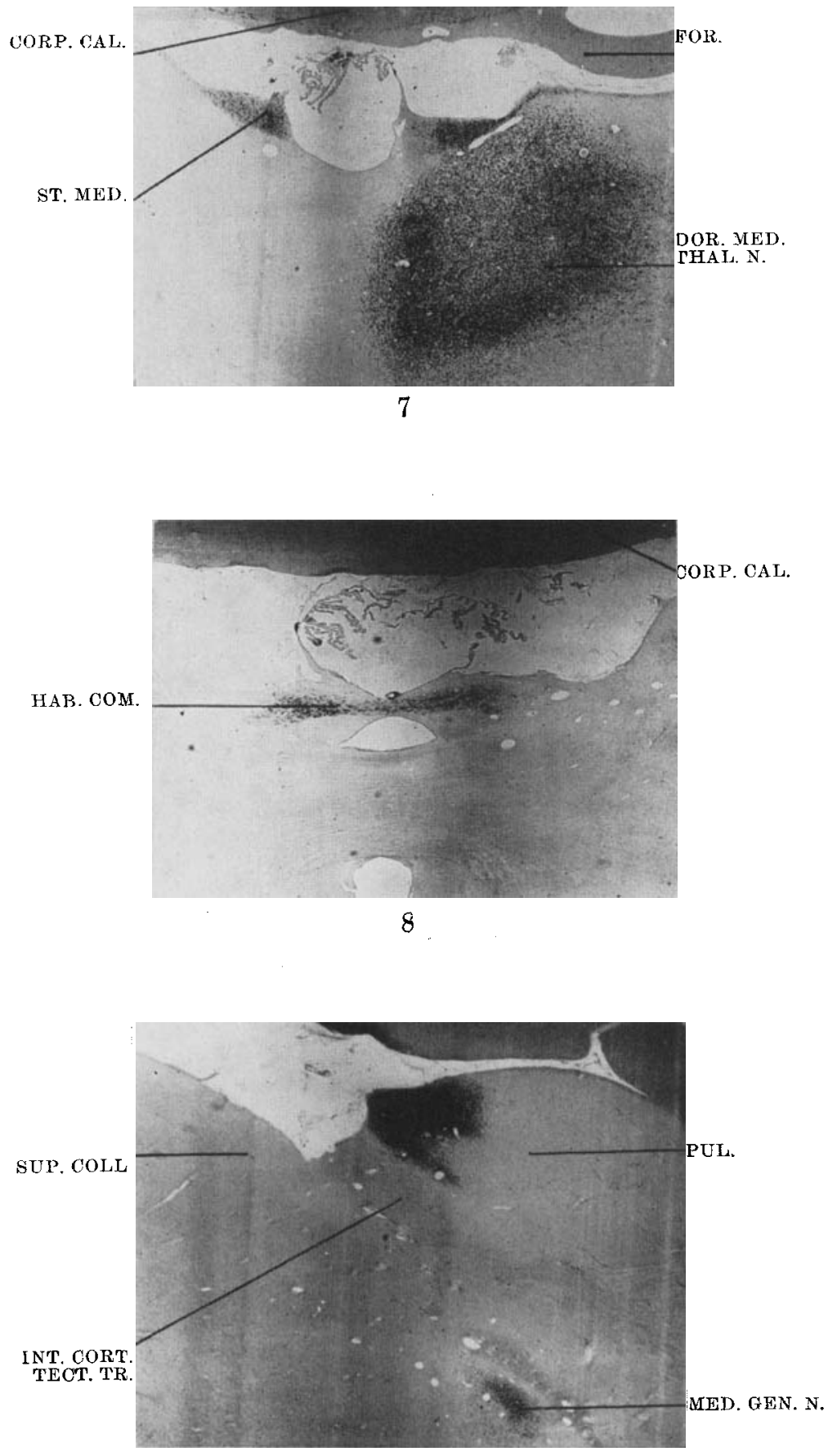


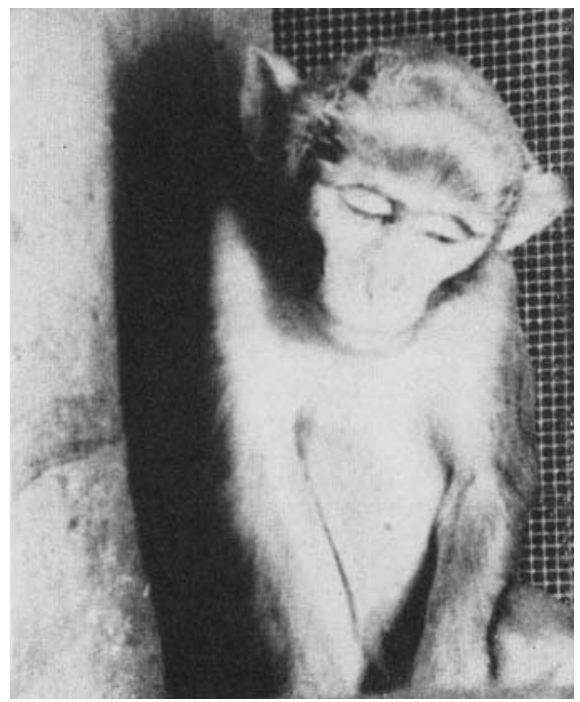

10

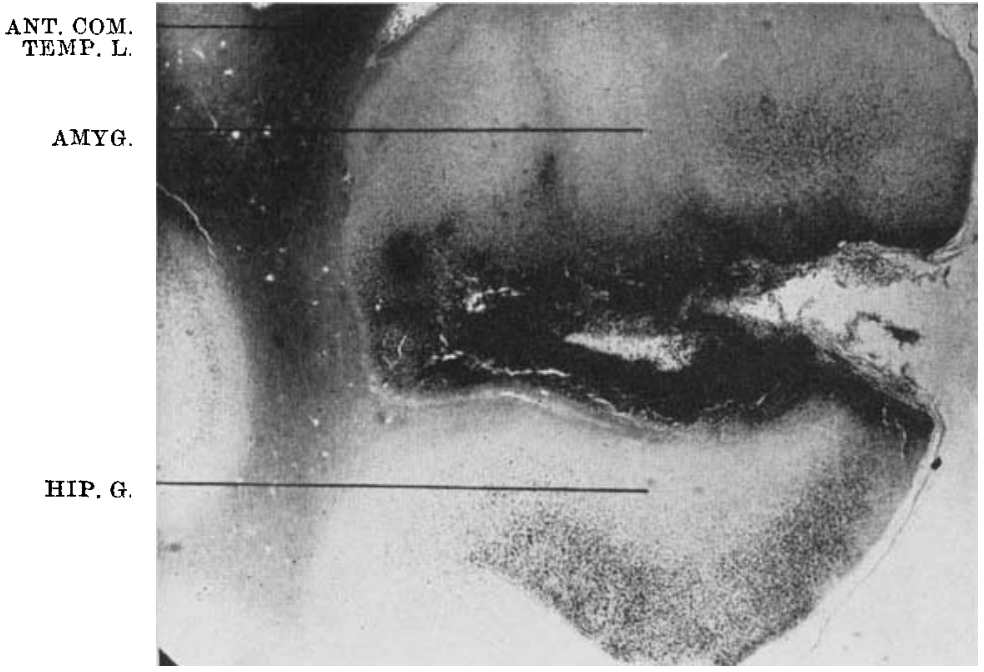

11 


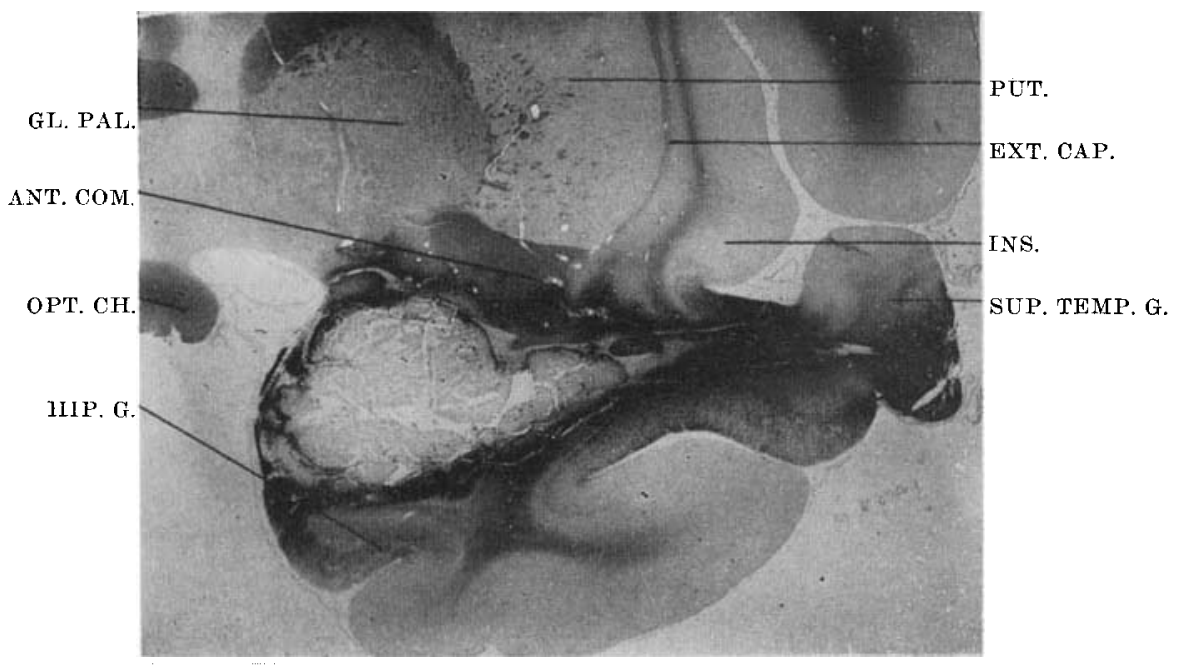

12

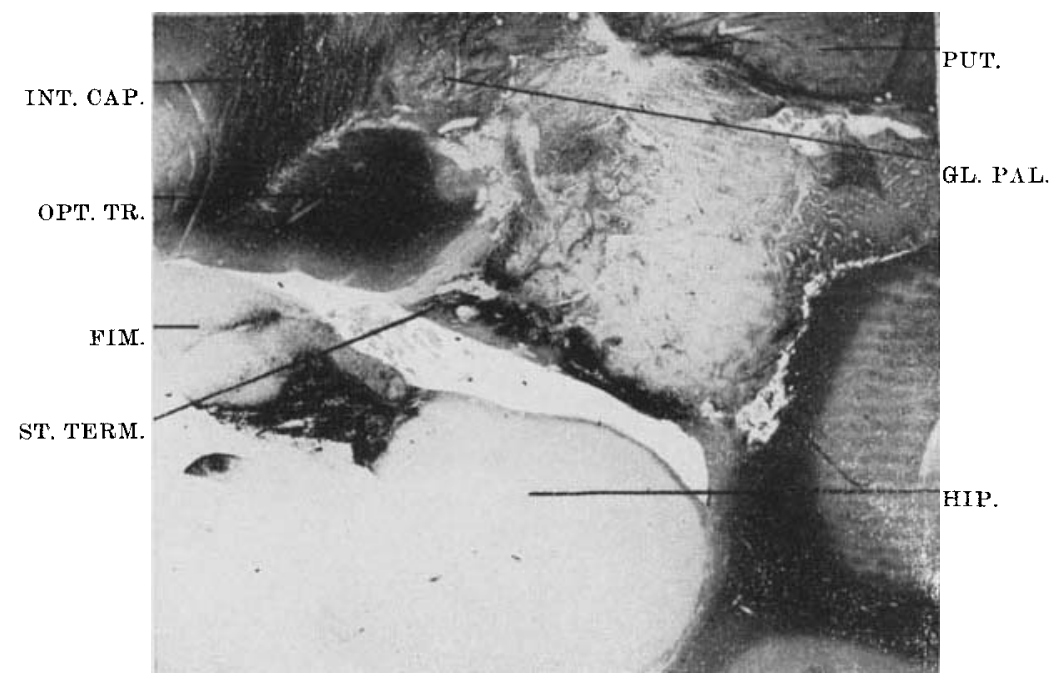




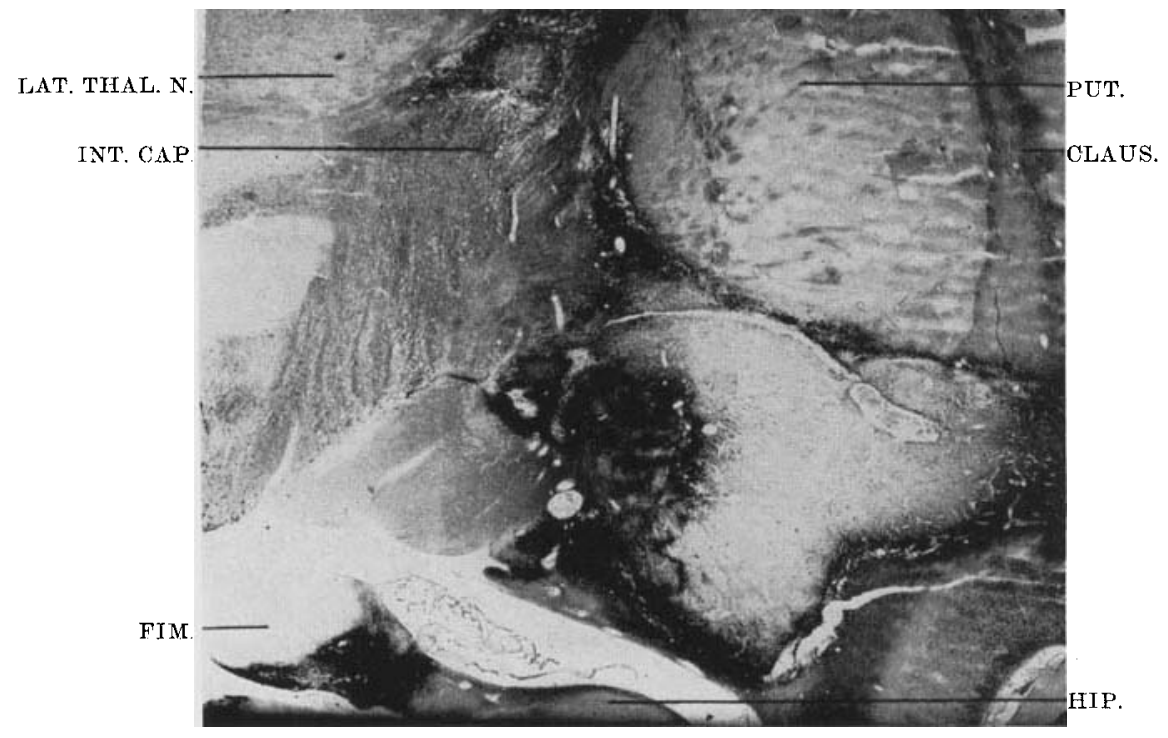

14

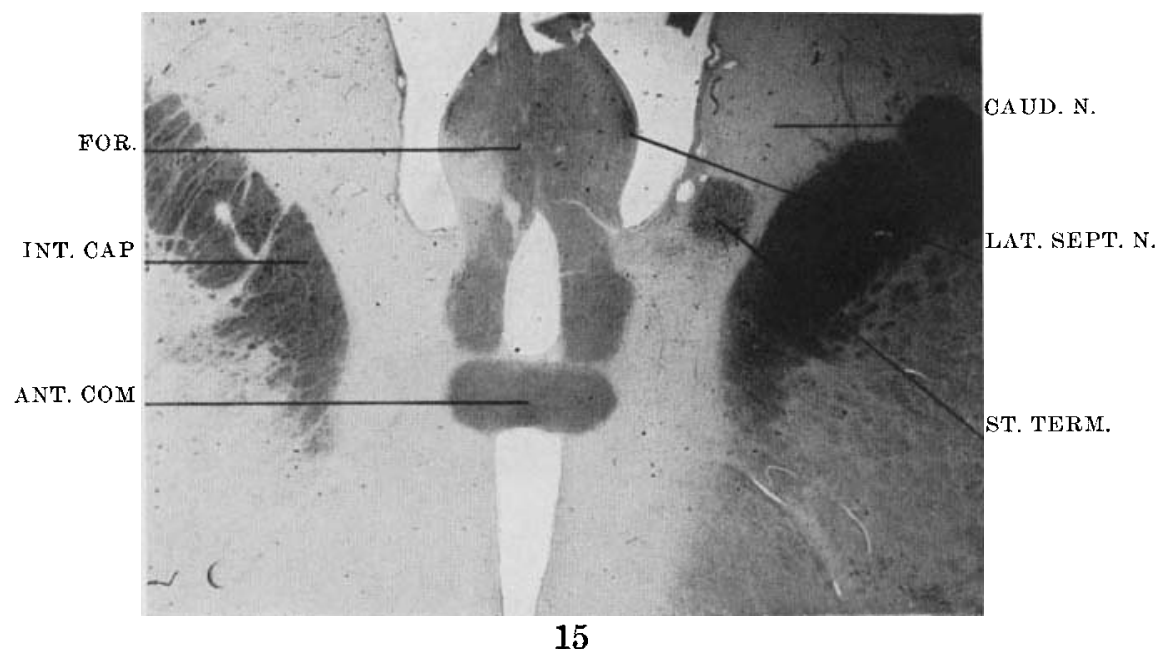




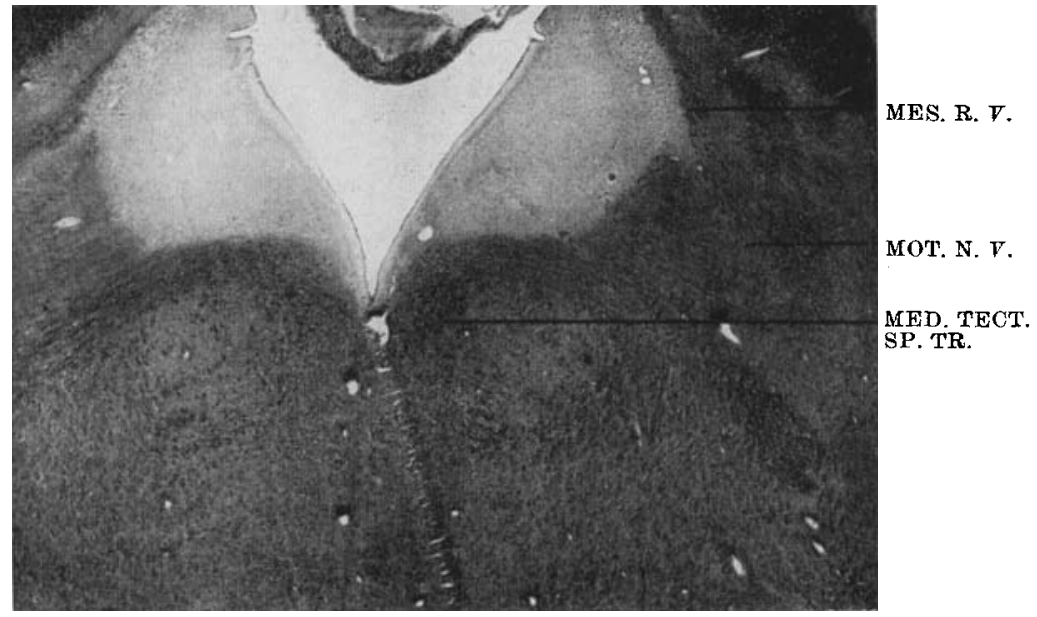

16

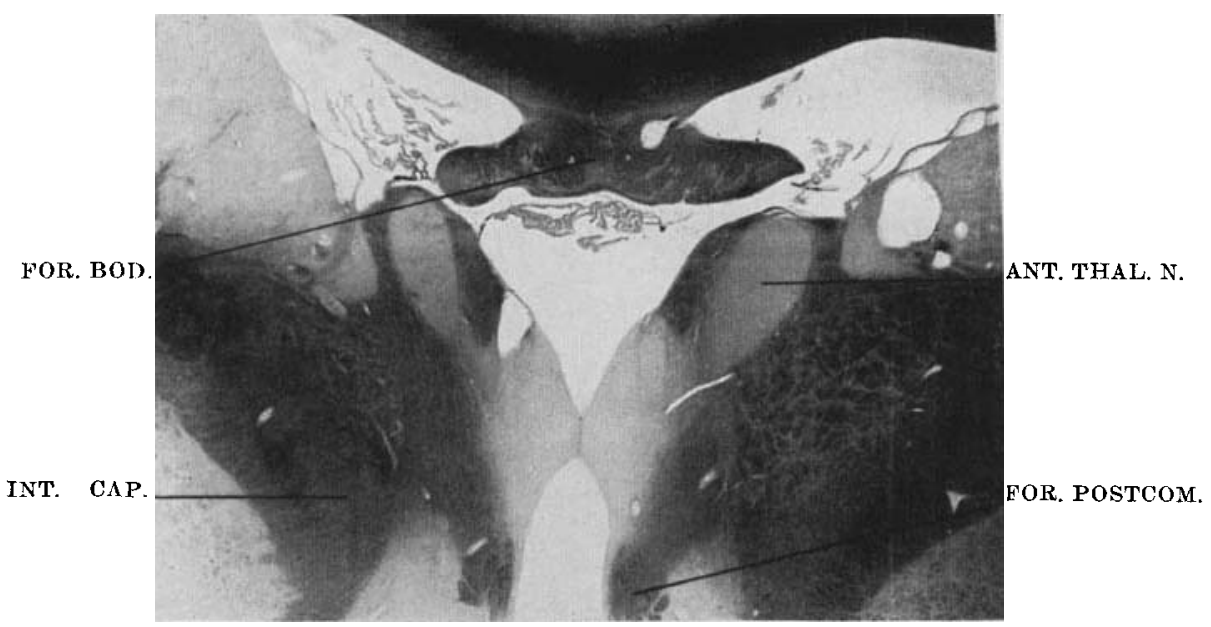

17 

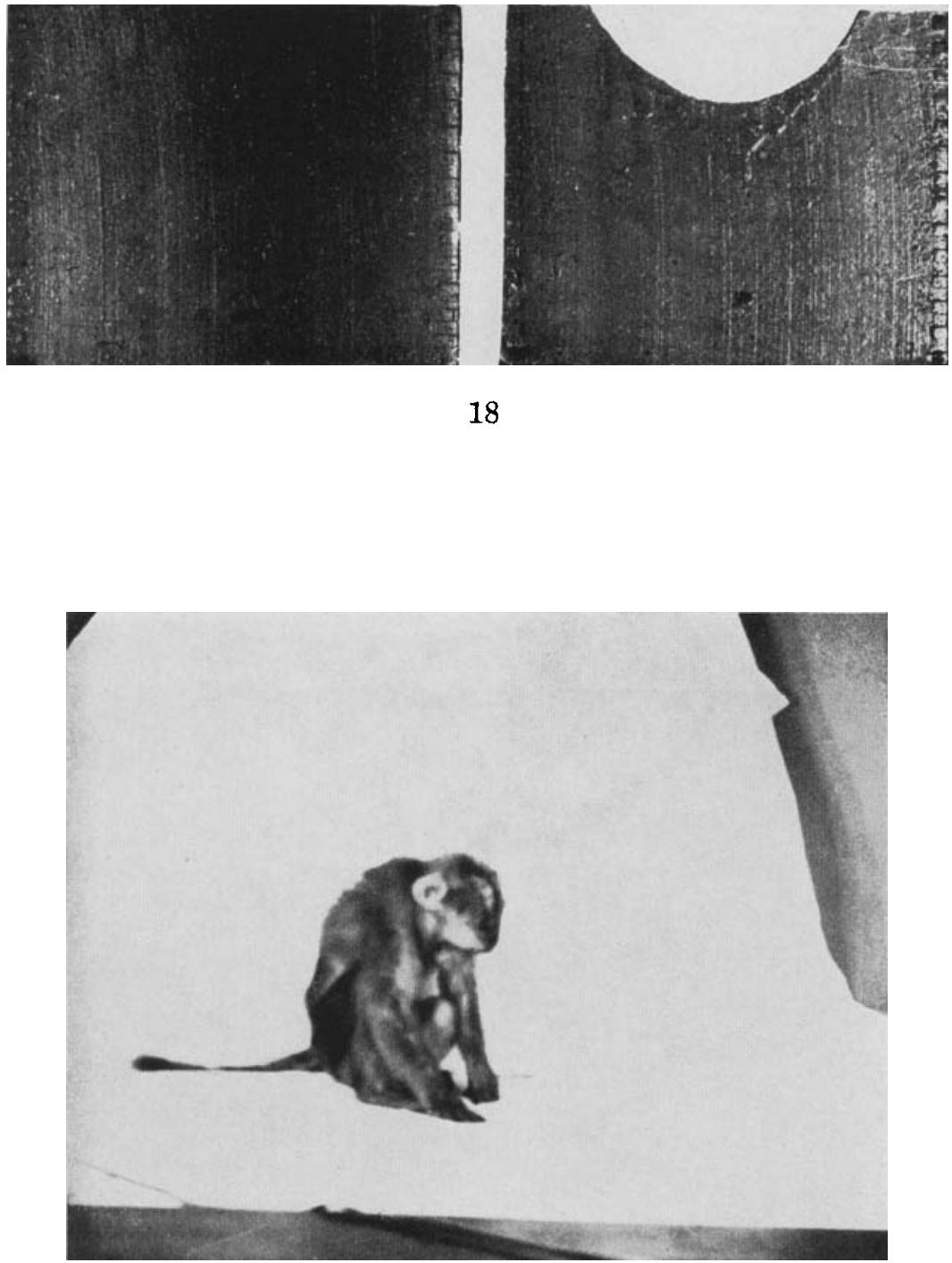